\title{
ON THE INDEX OF COMPOSITION OF THE EULER FUNCTION AND OF THE SUM OF DIVISORS FUNCTION
}

\author{
JEAN-MARIE DE KONINCK and FLORIAN LUCA ${ }^{\bowtie}$
}

(Received 17 January 2007; accepted 20 June 2007)

Communicated by I. E. Shparlinski

\begin{abstract}
Given an integer $n \geq 2$, let $\lambda(n):=(\log n) /(\log \gamma(n))$, where $\gamma(n)=\prod_{p \mid n} p$, denote the index of composition of $n$, with $\lambda(1)=1$. Letting $\phi$ and $\sigma$ stand for the Euler function and the sum of divisors function, we show that both $\lambda(\phi(n))$ and $\lambda(\sigma(n))$ have normal order 1 and mean value 1 . Given an arbitrary integer $k \geq 2$, we then study the size of $\min \{\lambda(\phi(n)), \lambda(\phi(n+1)), \ldots, \lambda(\phi(n+k-1))\}$ and of $\min \{\lambda(\sigma(n)), \lambda(\sigma(n+1)), \ldots, \lambda(\sigma(n+k-1))\}$ as $n$ becomes large.
\end{abstract}

2000 Mathematics subject classification: primary 11N25; secondary 11A25.

Keywords and phrases: Euler function, sum of divisors function.

\section{Introduction}

Given an integer $n \geq 2$, we define its index of composition by

$$
\lambda(n):=(\log n) /(\log \gamma(n)),
$$

where $\gamma(n)$ (often called the kernel of $n$ ) stands for the product of the distinct primes dividing $n$. For convenience, we let $\lambda(1)=\gamma(1)=1$. In a sense, $\lambda(n)$ measures the level of compositeness of $n$. First introduced by Browkin [2] in 2000, the function $\lambda$ was further studied by De Koninck and Doyon [3] who examined its global and local behavior, namely by showing that its mean value is 1 and moreover by establishing that given any integer $k \geq 2$ and setting

$$
Q_{k}(n):=\min \{\lambda(n), \lambda(n+1), \ldots, \lambda(n+k-1)\},
$$

and given any $\varepsilon>0$, then

$$
Q_{k}(n)>\frac{k}{k-1}-\varepsilon
$$

The first author was supported in part by a grant from NSERC. The second author was supported in part by Grants SEP-CONACyT 46755, PAPIIT IN104505 and a Guggenheim Fellowship.

(C) 2009 Australian Mathematical Society 1446-7887/2009 \$16.00 
for infinitely many values of $n$, which is most likely optimal. Indeed, De Koninck and Doyon [3, p. 164] conjecture that $\lim _{\sup _{n \rightarrow \infty}} Q_{k}(n)=k /(k-1)$ and show that the $a b c$ conjecture implies the validity of the above conjecture when $k=3$. In this paper, we show that the above conjecture from [3] holds under the $a b c$ conjecture for all $k \geq 2$.

More recently, De Koninck and Kátai [4] as well as De Koninck et al. [5] have studied the distribution function of $(\lambda(n)-1) \log n$ as $n$ runs through particular sets of integers, such as the shifted primes. The mean value of the function $\lambda(n)$ was also studied by Zhai [12].

In this paper, we also examine the global and local behavior of $\lambda(\phi(n))$ and $\lambda(\sigma(n))$, where $\phi$ and $\sigma$ stand for the Euler function and the sum of divisors function, respectively. More precisely, we first establish that each of $\lambda(\phi(n))$ and $\lambda(\sigma(n))$ have normal orders 1 and mean values 1 . Then, given an integer $k \geq 2$, we discuss the behavior of the expressions

$$
F_{k}(n):=\min \{\lambda(\phi(n)), \lambda(\phi(n+1)), \ldots, \lambda(\phi(n+k-1))\}
$$

and

$$
S_{k}(n):=\min \{\lambda(\sigma(n)), \lambda(\sigma(n+1)), \ldots, \lambda(\sigma(n+k-1))\}
$$

and conjecture that, for any fixed $k, F_{k}(n)$ and $S_{k}(n)$ can become arbitrarily large, providing heuristic arguments in their favor.

In what follows, the letter $p$ always stands for a prime number. Moreover, given any integer $n \geq 2$, let $P(n)$ stand for the largest prime factor of $n$. We shall also write $\omega(n)$ for the number of distinct prime factors of $n$ and $\Omega(n)$ for the total number of prime factors of $n$ counting their multiplicity, with $\omega(1)=\Omega(1)=0$. Finally, a positive integer $n$ is said to be powerful (or square-full) if $p^{2} \mid n$ whenever the prime number $p$ divides $n$.

We write $\log _{2} x$ for $\log \log x$ and we let $\log _{k} x=\log \log _{k-1} x$ for each integer $k \geq 3$. The input $x$ will always be assumed to be large enough so that the resulting iterated logarithms are greater than 1 .

We use the Landau symbols $O$ and $o$ as well as the Vinogradov symbols $\ll$ and $\gg$ with their usual meanings.

\section{Preliminary results}

Henceforth, given any integer $n \geq 2$, we shall write

$$
\phi(n)=A(n) B(n), \quad \text { with } \operatorname{gcd}(A(n), B(n))=1,
$$

where $A(n)$ is the square-full part of $\phi(n)$ and $B(n)$ its square-free part. To establish our results, we shall need the following lemmas.

LEMMA 1. As $x \rightarrow \infty$,

$$
\#\left\{n \leq x \mid \Omega(n)>10 \log _{2} x\right\}=O\left(\frac{x}{\log ^{2} x}\right) .
$$


PROOF. From [11, Lemma 13], uniformly for every positive integer $K$,

$$
\sum_{n \leq x: \Omega(n) \geq K} 1 \ll \frac{K}{2^{K}} x \log x .
$$

Applying this with $K=\left\lfloor 10 \log _{2} x\right\rfloor$ leads to the desired estimate.

LEMMA 2. The inequality $A(n) \leq(\log x)^{4}$ holds for all positive integers $n \leq x$ with $O\left(x /(\log x)^{2}\right)$ exceptions.

PROOF. It is well known that the number of square-full numbers $n \leq x$ is $O(\sqrt{x})$ (see, for example, [9, Theorem 14.4]). Given any $y \in[1, \sqrt{x}]$ and any square-full number $d \geq y$, it is clear that the number of positive integers $n \leq x$ that are multiples of $d$ is at most $x / d$, and therefore by Abel's summation formula, we easily get that the number of $n \leq x$ having a square-full divisor $d \geq y$ is $O(x / \sqrt{y})$. Taking $y=(\log x)^{4}$, we get the desired result.

LEMMA 3. For large $x$, the number of positive integers $n \leq x$ such that

$$
\max \{\Omega(\phi(n)), \Omega(\sigma(n))\}>110\left(\log _{2} x\right)^{2}
$$

is $O\left(x /(\log x)^{2}\right)$.

Proof. By Lemma 2, we may assume that $A(n)<(\log x)^{4}$. Thus,

$$
\phi(A(n)) \leq A(n) \leq \sigma(A(n))<(\log x)^{5},
$$

and therefore

$$
\max \{\Omega(\phi(A(n))), \Omega(\sigma(A(n)))\}<(5 / \log 2) \log \log x<10 \log _{2} x .
$$

By Lemma 1, we may further assume that $\Omega(B(n))<10 \log _{2} x$. Thus, if

$$
\max \{\Omega(\phi(n)), \Omega(\sigma(n))\}>110\left(\log _{2} x\right)^{2},
$$

it then follows that there exists a prime divisor $p$ of $n$ such that $\Omega(p \pm 1)>10 \log _{2} x$. Let $n=p m$. Then $p<x / m$, so that $p \pm 1 \leq x / m+1 \leq 2 x / m$. The number of such numbers $p$ is, by the argument from the proof of Lemma 1 , at most a multiple of

$$
\frac{K}{2^{K}} \frac{x \log x}{m},
$$

where $K=\left\lfloor 10 \log _{2} x\right\rfloor$. Summing up over all values of $m \leq x$, the number of such numbers $n \leq x$ is at most

$$
\frac{K \log x}{2^{K}} \sum_{m \leq x} \frac{1}{m} \ll \frac{x(\log x)^{2} \log _{2} x}{2^{\left\lfloor 10 \log _{2} x\right\rfloor}} \ll \frac{x}{(\log x)^{2}},
$$

because $10 \log 2>4$. 
LEMMA 4. The estimate

$$
\#\left\{n \leq x: p^{2} \mid \sigma(n) \text { for some } p>(\log x)^{5}\right\}=O\left(\frac{x}{(\log x)^{2}}\right)
$$

holds as $x \rightarrow \infty$. A similar estimate holds when $\sigma(n)$ is replaced by $\phi(n)$.

Proof. By Lemma 2, we may assume that $A(n)<(\log x)^{4}$. Hence,

$$
\phi(A(n)) \leq A(n) \leq \sigma(A(n))<(\log x)^{5}
$$

for large $x$. If $p^{2} \mid \sigma(n)$ or $p^{2} \mid \phi(n)$ for some $p>(\log x)^{5}$, it follows that $p^{2} \mid$ $\sigma(B(n))$ or $p^{2} \mid \phi(B(n))$, respectively. Now [1, Lemma 2] shows that

$$
\#\left\{n \leq x \mid \phi(B(n)) \equiv 0 \bmod p^{2}\right\} \ll \frac{x\left(\log _{2} x\right)^{2}}{p^{2}},
$$

and a straightforward adaptation of it shows that the same is true when $\phi$ is replaced by $\sigma$. Thus, the number of positive integers $n \leq x$ such that either $p^{2} \mid \sigma(n)$ or $p^{2} \mid \phi(n)$ for some $p>(\log x)^{5}$ is, by the above inequality, at most a multiple of

$$
x\left(\log _{2} x\right)^{2} \sum_{p>(\log x)^{5}} \frac{1}{p^{2}}<x\left(\log _{2} x\right)^{2} \int_{(\log x)^{5}}^{x^{1 / 2}} \frac{d t}{t^{2}} \ll \frac{x\left(\log _{2} x\right)^{2}}{(\log x)^{5}} \ll \frac{x}{(\log x)^{2}} .
$$

\section{The normal order of $\lambda(\phi(n))$}

Here, we prove the following result.

THEOREM 5. For every $\varepsilon>0$, the inequality $1 \leq \lambda(\phi(n)) \leq 1+\varepsilon$ holds for all $n$ except for a set of asymptotic density zero. The same inequality holds when $\phi$ is replaced by $\sigma$.

PROOF. We shall prove this result only for $\sigma$ since the proof for $\phi$ is entirely similar. Since $n \leq \sigma(n) \ll n \log _{2} n$ holds for all $n$, we have that

$$
\log (\sigma(n))=\log n+O\left(\log _{3} n\right) .
$$

By Lemmas 2-4, for most $n$ we have that if $Q(n)$ is the largest prime $p$ such that $p^{2} \mid \sigma(n)$ (equivalently, $\left.Q(n)=P(\sigma(n) / \gamma(\sigma(n)))\right)$, then $Q(n)<(\log n)^{5}$. Furthermore, $\Omega(\sigma(n))<110\left(\log _{2} n\right)^{2}$. This shows that

$$
\log (\gamma(\sigma(n))) \geq \log (\sigma(n))-\Omega(\sigma(n)) \log (Q(n))=\log n+O\left(\left(\log _{2} n\right)^{3}\right) .
$$

From estimates (6) and (7), we immediately get that for most $n$,

$$
\lambda(\sigma(n))=1+O\left(\frac{\left(\log _{2} n\right)^{3}}{\log n}\right)=1+o(1), \quad \text { as } n \rightarrow \infty,
$$

which is what we wanted to prove. 


\section{The mean value of $\lambda(\phi(n))$}

In this section we prove the following result.

THEOREM 6. The estimate

$$
\frac{1}{x} \sum_{n \leq x} \lambda(\phi(n))=1+o(1)
$$

holds as $x \rightarrow \infty$. The same holds when $\phi$ is replaced by $\sigma$.

PROOF. Again, we shall give the proof only for $\sigma$ since for $\phi$ it is entirely similar. The arguments from Section 3 show that the estimates

$$
\log (\sigma(n))=\log x+O\left(\log _{3} x\right) \quad \text { and } \quad \log (\gamma(\sigma(n)))=\log x+O\left(\left(\log _{2} x\right)^{3}\right)
$$

both hold for all positive integers $n \leq x$ with at most $O\left(x /(\log x)^{2}\right)$ exceptions. On the exceptional set, it is clear that $\lambda(\sigma(n)) \leq \log x$. Hence,

$$
\begin{aligned}
\sum_{n \leq x} \lambda(\sigma(n)) & =\sum_{\substack{n \leq x: Q(n)<(\log x)^{5} \\
\Omega(\sigma(n))<110\left(\log _{2} x\right)^{2}}}\left(1+O\left(\frac{\left(\log _{2} x\right)^{3}}{\log x}\right)\right)+O\left(\frac{x}{(\log x)^{2}} \log x\right) \\
& =x+O\left(\frac{x\left(\log _{2} x\right)^{3}}{\log x}\right),
\end{aligned}
$$

which is the desired estimate.

\section{The local behavior of $\lambda(\phi(n))$}

We prove the analogue of [3, Theorem 3] for the case of the quantity $F_{k}(n)$ given by (3).

THEOREM 7. Given any integer $k \geq 2$, for every $\varepsilon>0$, there exist infinitely many $n$ such that

$$
F_{k}(n)>\frac{k}{k-1}-\varepsilon .
$$

PROOF. We follow the method of [3, Proof of Theorem 3]. Let $y>k$ be sufficiently large so that the interval $\left[y, y+y^{2 / 3}\right]$ contains at least $k$ prime numbers. Let these be $y<p_{1}<\cdots<p_{k}<y+y^{2 / 3}$. Observe that

$$
\frac{p_{k}}{p_{1}}=1+O\left(\frac{1}{y^{1 / 3}}\right)=1+o(1) \quad(y \rightarrow \infty) .
$$

Let $a>3$ be a large positive integer and let $n$ be such that $n \equiv-i \bmod p_{i}^{a}$ for all $i=1,2, \ldots, k$. This system is solvable by the Chinese remainder theorem and it therefore has a solution $n \in[M, 2 M)$, where $M=\prod_{i=1}^{k} p_{i}^{a}$. Since

$$
2 M+O(1) \geq n+i>\phi(n+i) \gg \frac{n+i}{\log _{2}(n+i)} \geq \frac{M}{\log _{2}(2 M+k)},
$$

we get that 


$$
\log (\phi(n+i))=n+i+O\left(\log _{3} M\right)=\log M+O\left(\log _{3} M\right), \quad i=1,2, \ldots, k,
$$

whenever the $p_{i}$ are fixed and $a$ tends to infinity. However, note that since $n+i=$ $p_{i}^{a} m_{i}$ for some positive integer $m_{i}$,

$$
\phi(n+i)=p_{i}^{a-1}\left(p_{i}-1\right) n_{i},
$$

for some positive integer $n_{i}$ (here, $n_{i}=\phi\left(m_{i}\right)$ if $p_{i} \nmid m_{i}$ and $n_{i}=\phi\left(m_{i}\right) p_{i} /\left(p_{i}-1\right)$ if $p_{i} \mid m_{i}$, so that in any case $n_{i} \leq m_{i}$ always holds). Therefore, in light of (9), for each $i=1,2, \ldots, k$,

$$
\begin{aligned}
\log (\gamma(\phi(n+i))) & \leq \log \left(p_{i} \gamma\left(p_{i}-1\right) \gamma\left(n_{i}\right)\right) \leq \log \left(p_{i}^{2} m_{i}\right) \\
& =\log \left(\frac{p_{i}^{a} m_{i}}{p_{i}^{a-2}}\right)=\log \left(\frac{n+i}{p_{i}^{a-2}}\right) \\
& =\log (\phi(n+i))+O\left(\log _{3} M\right)-(a-2) \log p_{i} \\
& =\log M+O\left(\log _{3} M\right)-(a-2) \log p_{i} .
\end{aligned}
$$

On the other hand, using (8), it is clear that

$$
\log M=a \sum_{j=1}^{k} \log p_{j}=k a(1+o(1)) \log p_{i}, \quad i=1,2, \ldots, k .
$$

Combining (10) and (11), we obtain that

$$
\begin{aligned}
\log (\gamma(\phi(n+i))) & \leq \log M-\frac{\log M}{k}(1+o(1))+O\left(\log _{3} M\right) \\
& =\left(1-\frac{1}{k}+o(1)\right) \log M,
\end{aligned}
$$

which together with estimate (9) shows that, for each $i=1,2, \ldots, k$,

$$
\lambda(\phi(n+i))=\frac{\log (\phi(n+i))}{\log (\gamma(\phi(n+i)))} \geq \frac{1}{1-(1 / k)+o(1)}=\frac{k}{k-1}+o(1),
$$

which implies the desired inequality.

\section{The local behavior of $\lambda(\sigma(n))$}

Here, the method of proof of Theorem 7 does not work because if $p$ is a fixed prime and $a$ is a positive integer, then $\gamma\left(\sigma\left(p^{a}\right)\right)$ is not small (in fact, it probably tends to infinity with $a$, and the $a b c$ conjecture predicts that it is as large as $p^{a(1-\varepsilon)}$ for every $\varepsilon>0$ provided that $a$ is sufficiently large with respect to $\varepsilon$ ). However, the same result holds nevertheless. 
THEOREM 8. Given any integer $k \geq 2$, for every $\varepsilon>0$, the inequality

$$
S_{k}(n) \geq \frac{k}{k-1}-\varepsilon
$$

holds for infinitely many positive integers $n$.

We shall need the following well-known lemma, essentially due to Erdős [7].

LEMMA 9. There exists a constant $\delta \in(0,1)$ such that the estimate

$$
\#\left\{p \in[y, 2 y] \mid P(p+1)<y^{\delta}\right\} \gg \pi(y)
$$

holds for large $y$.

Specific values of $\delta$ are known from the work of several mathematicians but they are of no use to us.

Proof. Let $\delta \in(0,1)$ be as in Lemma 9, $y$ be large and $\varepsilon \in(0,1-\delta)$. Let $U=\left\lfloor y^{\delta+\varepsilon}\right\rfloor$ and $V=k U$. Choose $p_{1}<\cdots<p_{V}$ primes in $(y, 2 y)$ such that

$$
P\left(p_{i}+1\right)<y^{\delta} \quad \text { for all } i=1,2, \ldots, V .
$$

This is possible for large $y$ by Lemma 9 and the fact that $V=O\left(y^{\delta+\varepsilon}\right)=o(\pi(y))$ as $y \rightarrow \infty$. For $j=1,2, \ldots, k$ put

$$
m_{j}=\prod_{i=U(j-1)+1}^{U j} p_{i}
$$

Note that

$$
\log m_{j}=\sum_{i=U(j-1)+1}^{U j} \log p_{i}=U \log y+O(U)=(1+o(1)) y^{\delta+\varepsilon} \log y
$$

for all $j=1,2, \ldots, k$ as $y \rightarrow \infty$. Since $\sigma\left(m_{j}\right)=\prod_{p \mid m_{j}}(p+1)$, it follows, from the way we have chosen the prime factors of $m_{j}$, that

$$
\gamma\left(\sigma\left(m_{j}\right)\right) \leq \prod_{p \leq y^{\delta}} p=\exp \left((1+o(1)) y^{\delta}\right),
$$

where the last estimate follows from the prime number theorem. Therefore

$$
\log \gamma\left(\sigma\left(m_{j}\right)\right) \leq(1+o(1)) y^{\delta}=o\left(\log \left(m_{j}\right)\right)
$$

for all $j=1,2, \ldots, k$ as $y \rightarrow \infty$. Now let $n$ be a positive integer such that $n+j \equiv 0 \bmod m_{j}$ for all $j=1,2, \ldots, k$. The above system is solvable by the Chinese remainder theorem and all its solutions are of the form $n=M \ell+N$, where $M=\prod_{j=1}^{k} m_{j}$ and $N \in[0,1, \ldots, M-1]$ is the smallest nonnegative solution of 
the above system of congruences. We claim that there exists $\ell \in[y, 2 y]$ such that the corresponding $n$ satisfies the fact that $(n+j) / m_{j}$ and $m_{j}$ are coprime for $j=1,2, \ldots, k$. Indeed, note that

$$
(n+j)=M \ell+(N+j)=m_{j}\left(\left(M / m_{j}\right) \ell+(N+j) / m_{j}\right),
$$

so that

$$
(n+j) / m_{j}=\left(M / m_{j}\right) \ell+(N+j) / m_{j} .
$$

Clearly, $M / m_{j}$ and $m_{j}$ are coprime since $M$ is square-free. Thus, if $(n+j) / m_{j}$ and $m_{j}$ are, say, both divisible by the prime $p$, then this puts $\ell$ into a certain uniquely determined congruence class modulo $p$. The number of such $\ell$ in the interval $[y, 2 y]$ is less than or equal to $y / p+1$. Thus, the number of $\ell \in[y, 2 y]$ for which the corresponding $n$ has the property that $(n+j) / m_{j}$ and $m_{j}$ are not coprime for some $j=1,2, \ldots, k$ is at most

$$
y \sum_{p \mid M} \frac{1}{p}+\omega(M) \leq \frac{k y^{1+\delta+\varepsilon}}{y}+k y^{\delta+\varepsilon}<2 k y^{\delta+\varepsilon} .
$$

Since $\delta+\varepsilon<1$ and since the interval $[y, 2 y]$ contains at least $y-1$ integers, we get that there are at least $y-1-2 k y^{\delta+\varepsilon}>0$ integers $\ell \in[y, 2 y]$ such that the corresponding $n$ does indeed have the property that $(n+j) / m_{j}$ and $m_{j}$ are coprime for all $j=1,2, \ldots, k$. Such an $n$ has the following properties:

$$
\begin{aligned}
\log (\sigma(n+j)) & =(1+o(1)) \log n=(1+o(1))(\log M+\log y) \\
& =(k+o(1)) y^{\delta+\varepsilon} \log y ;
\end{aligned}
$$

further, since $(n+j) / m_{j}$ and $m_{j}$ are coprime,

$$
\sigma(n+j)=\sigma\left(m_{j}\right) \sigma\left((n+j) / m_{j}\right),
$$

so that

$$
\begin{aligned}
\log (\gamma(\sigma(n+j))) & \leq \log \left(\gamma\left(\sigma\left(m_{j}\right)\right)\right)+\log \left(\gamma\left(\sigma\left((n+j) / m_{j}\right)\right)\right) \\
& =o\left(\log \left(m_{j}\right)\right)+(1+o(1)) \log \left((n+j) / m_{j}\right) \\
& =(1+o(1))\left(\log n-\log m_{j}\right) \\
& =(1+o(1))\left(\log M+\log y-\log m_{j}\right) \\
& =(k-1+o(1)) y^{\delta+\varepsilon} \log y,
\end{aligned}
$$

which yields

$$
\lambda(\sigma(n+j)) \geq \frac{k}{k-1}+o(1)
$$

for all $j=1,2, \ldots, k$ as $y \rightarrow \infty$, therefore establishing the desired conclusion. 


\section{Heuristics}

As we have already mentioned, [3, Theorem 3] shows that inequality (2) holds for infinitely many $n$, and it was conjectured that apart from the $\varepsilon$ this inequality is the best possible. Here, we prove that this is indeed so under the $a b c$ conjecture.

THEOREM 10. For each integer $k \geq 2$, let $Q_{k}(n)$ be as in (1). The estimate

$$
\limsup _{n \rightarrow \infty} Q_{k}(n)=\frac{k}{k-1}
$$

holds under the abc conjecture.

PROOF. Instead of recalling the $a b c$ conjecture, we recall the following consequence of it (see [6, 8], or [10]).

LEMMA 11 (The ABC conjecture). Let $f$ be a homogeneous polynomial with integer coefficients having no repeated irreducible factors. Then for every $\varepsilon>0$ and coprime positive integers $m$ and $n$,

$$
\gamma(f(m, n)) \gg \max \{m, n\}^{d-2-\varepsilon},
$$

where $d$ is the degree of $f$ and the constant implied by the Vinogradov symbol above depends on both $f$ and $\varepsilon$.

The classical $a b c$ conjecture is usually the above statement for the polynomial $f(X, Y)=X Y(X+Y)$. To deduce Theorem 10 from Lemma 11, we may assume that $k \geq 3$ and look at the homogeneous polynomial

$$
f(X, Y)=X Y(Y-X)(2 Y-X)(3 Y-2 X) \ldots((k-1) Y-(k-2) X),
$$

which obviously has degree $k+1$ and no repeated factors. Note that

$$
f(n, n+1)=n(n+1)(n+2)(n+3) \ldots(n+k-1),
$$

so that by Lemma 11 we have that the inequality

$$
\gamma(n(n+1) \ldots(n+k-1)) \gg n^{k-1-\varepsilon / 2}
$$

holds for every fixed $\varepsilon>0$ where the implied constant depends on $\varepsilon$ and $k$. Now consider an integer $n$ such that

$$
Q_{k}(n) \geq \frac{k}{k-1}+\varepsilon .
$$

Then

$$
\gamma(n+i) \leq(n+i)^{((k-1) /(k+(k-1) \varepsilon))} \ll n^{((k-1) /(k+(k-1) \varepsilon))}, \quad i=0,1, \ldots, k .
$$


Multiplying all these relations for $i=0,1, \ldots, k-1$, we get that

$$
\prod_{i=1}^{k} \gamma(n+i-1) \ll n^{((k(k-1)) /(k+(k-1) \varepsilon))} .
$$

But for $\varepsilon<1 /(k-1)$,

$$
\frac{k(k-1)}{k+(k-1) \varepsilon}<k-1-\varepsilon
$$

because this last inequality is equivalent to $(k-1)^{2} \geq k+(k-1) \varepsilon$, which is implied by $(k-1)^{2} \geq k+1$ (because $\varepsilon \leq 1 /(k-1)$ ), and this last inequality is equivalent to $k \geq 3$. Hence,

$$
\gamma(n(n+1) \ldots(n+k-1)) \leq \prod_{i=1}^{k} \gamma(n+i-1) \ll n^{k-1-\varepsilon},
$$

which compared with inequality (12) gives us an upper bound on $n$. This completes the proof of the theorem.

We conjecture that, unlike $Q_{k}(n)$, both the amounts $F_{k}(n)$ and $S_{k}(n)$ should be unbounded and that in fact each of the inequalities $F_{k}(n) \gg \log n$ and $S_{k}(n) \gg \log n$ should hold for infinitely many positive integers $n$, where the implied constants depend on $k$. In what follows, we will treat only the case of $F_{k}(n)$. To see why, let us first look at the case $k=2$.

If there existed infinitely many primes $p$ of the form $2^{a} \cdot 3^{b}+1$, then it would follow that $F_{2}(n)$ is unbounded. Indeed, let $p=2^{a} \cdot 3^{b}+1$ be such a large prime and set $n=p-1$. Then

$$
\phi(n)=\phi\left(2^{a} \cdot 3^{b}\right)=2^{a} \cdot 3^{b-1} \text { and } \phi(n+1)=2^{a} \cdot 3^{b},
$$

so that $\lambda(\phi(n))=((a \log 2+(b-1) \log 3) /(\log 2+\log 3)) \gg \log n$ and similarly $\lambda(\phi(n+1)) \gg \log n$. Hence, $F_{2}(n) \gg \log n$, proving our claim. A computer check showed that the number of primes $p \leq x$ of the above form is equal to 66 for $x=10^{10}$ and to 789 for $x=10^{100}$.

Using essentially the same argument as above, let us show how one would go about constructing integers $n$ for which $F_{k}(n) \gg \log n$. Assume that

$$
2=p_{1}<p_{2}<\cdots<p_{k}
$$

are the first $k$ prime numbers. Assume that $a_{1}, \ldots, a_{k}$ are such that $a_{i}>\log k / \log p_{i}$ and such that if we set

$$
n=p_{1}^{a_{1}} p_{2}^{a_{2}} \ldots p_{k}^{a_{k}}
$$


then $(n+i) / i$ is a prime number for all $i=1,2, \ldots, k$. Note that, from the conditions we imposed on the exponents $a_{i}$, the number $(n+i) / i$ is always an integer coprime to $i$. If this is the case, then

$$
\phi(n+i)=\phi(i)\left(\frac{n+i}{i}-1\right)=\frac{\phi(i) n}{i},
$$

so that

$$
\gamma(\phi(n+i))=\log \left(p_{1} \ldots p_{k}\right)=O(1) \text { for all } i=1,2, \ldots, k \text {. }
$$

Thus

$$
\lambda(\phi(n+i)) \gg \log (\phi(n+i)) \gg \log n
$$

for all such choices of $n$.

To back up our construction a little more, we give heuristic support to the existence of infinitely many positive integers $n$ of the above form. Let $X$ be a large positive integer. There are at least a multiple of $X^{k} k$-tuples of integers $\left(a_{1}, \ldots, a_{k}\right)$ such that $\left(a_{1}, \ldots, a_{k}\right) \in(X, 2 X)^{k}$. For each one of them, we assume, heuristically, that the probability of each one of the numbers $(n+i) / i$ being prime is roughly

$$
1 / \log ((n+i) / i) \gg 1 / X \text {. }
$$

Of course, this cannot possibly be true for all such $k$-tuples $\left(a_{1}, \ldots, a_{k}\right)$ because the number $n / i$ might end up having all exponents divisible by the same odd prime in which case the expression $n / i+1$ factors in an obvious way. To fix this, we may first fix $a_{1}, \ldots, a_{k-1}$ in an arbitrary manner, and then fix $a_{k}$ to be any prime in $(X, 2 X)$ which does not divide any of $a_{i}$ for $i=1,2, \ldots, k-1$ (note that if $X$ is large, $a_{k}$ can be any prime in $(X, 2 X)$ except for at most $k-1$ of them). Assuming further that the events that $(n+i) / i$ are prime are independent for $i=1,2, \ldots, k$, we conclude that if $X$ is large, for a suitable set of choices of $\left(a_{1}, \ldots, a_{k}\right) \in(X, 2 X)^{k}$ of total cardinality at least a multiple of

$$
X^{k-1}(\pi(2 X)-\pi(X)-k+1) \gg X^{k} / \log X,
$$

the probability that all numbers $(n+i) / i$ are simultaneously prime is at least a multiple of $1 / X^{k}$. Multiplying those two amounts, we get that the expected number of such primes is at least a multiple of $1 / \log X$. Now letting $X=2^{\ell}$ go to infinity through powers of 2 starting with a sufficiently large $2^{\ell_{0}}$, we get that the number of such numbers $n$ should be at least a multiple of $\sum_{\ell \geq \ell_{0}} 1 / \ell$, hence, an infinite number of them.

Computationally, letting $k=4$ and choosing

$$
n=2^{8} \cdot 3^{30} \cdot 5^{20}=5026638967154516601562500000000,
$$


TABLE 1 . Some values of $F_{k}(n)$.

\begin{tabular}{llcc}
\hline$k$ & $n$ & Number of digits of $n$ & $\left\lfloor F_{k}(n)\right\rfloor$ \\
\hline 2 & $2^{44} \cdot 3^{40}$ & 33 & 40 \\
2 & $2^{491} \cdot 3^{579}$ & 425 & 544 \\
3 & $2^{77} \cdot 3^{213}$ & 125 & 159 \\
4 & $2^{43} \cdot 3 \cdot 5^{7}$ & 19 & 17 \\
4 & $2^{8} \cdot 3^{30} \cdot 5^{20}$ & 31 & 20 \\
4 & $2^{12} \cdot 3^{29} \cdot 5^{281}$ & 214 & 144 \\
5 & $2^{46} \cdot 3^{41} \cdot 5^{19}$ & 47 & 31 \\
6 & $2^{42} \cdot 3^{6} \cdot 5^{5} \cdot 7^{4} \cdot 13^{24}$ & 58 & 16 \\
\hline
\end{tabular}

one can check that $n+1,(n+2) / 2$ and $(n+3) / 3$ are all prime numbers. This allows us to obtain that

$$
\begin{aligned}
\phi(n)= & 2^{10} \cdot 3^{29} \cdot 5^{19}, \\
& \text { so that } \lambda(\phi(n))=\frac{10 \log 2+29 \log 3+19 \log 5}{\log 2+\log 3+\log 5} \approx 20.3959, \\
\phi(n+1)= & 2^{8} \cdot 3^{30} \cdot 5^{20}, \\
& \text { so that } \lambda(\phi(n+1))=\frac{8 \log 2+30 \log 3+20 \log 5}{\log 2+\log 3+\log 5} \approx 20.7845, \\
\phi(n+2)= & \phi\left(2^{8} \cdot 3^{30} \cdot 5^{20}+2\right)=\phi\left(2\left(2^{7} \cdot 3^{30} \cdot 5^{20}+1\right)\right)=2^{7} \cdot 3^{30} \cdot 5^{20}, \\
& \text { so that } \lambda(\phi(n+2))=\frac{7 \log 2+30 \log 3+20 \log 5}{\log 2+\log 3+\log 5} \approx 20.5807, \\
\phi(n+3)= & \phi\left(2^{8} \cdot 3^{30} \cdot 5^{20}+3\right)=\phi\left(3\left(2^{8} \cdot 3^{29} \cdot 5^{20}+1\right)\right)=2 \cdot 2^{8} \cdot 3^{29} \cdot 5^{20}, \\
& \text { so that } \lambda(\phi(n+3))=\frac{9 \log 2+29 \log 3+20 \log 5}{\log 2+\log 3+\log 5} \approx 20.6653,
\end{aligned}
$$

thus establishing that

$$
F_{4}(n) \approx 20.3959=\min (20.3959,20.7845,20.5807,20.6653) .
$$

More examples can be seen in Table 1.

As mentioned above, similar heuristics apply for $S_{k}(n)$. In fact, if instead one does not start with only the first $k$ primes $2=p_{1}<\cdots<p_{k}$, but with the first $2 k$ primes and sets $n=p_{1}^{a_{1}} p_{2}^{a_{2}} \ldots p_{2 k}^{a_{2 k}}$ for some sufficiently large positive integers $a_{i}$ with $i=1,2, \ldots, 2 k$, then one can further assume that $(n+i) / n$ and $(n-i) / n$ are both primes for all $i=1,2, \ldots, k$, and then with such $n$ one finds that the even 
stronger inequality $\min \left\{F_{k}(n), S_{k}(n)\right\} \gg \log n$ holds. We let the reader fill in the details of such a deduction as well as working out a heuristic that would predict that there should indeed be infinitely many such positive integers $n$.

\section{Acknowledgments}

The authors thank the anonymous referee and Professor Igor Shparlinski for helpful suggestions.

\section{References}

[1] N. L. Bassily, I. Kátai and M. Wijsmuller, 'On the prime power divisors of the iterates of the Euler- $\phi$ function', Publ. Math. Debrecen 55(1-2) (1999), 17-32.

[2] J. Browkin, 'The $a b c$-conjecture', in: Number Theory, Trends in Mathematics (Birkhäuser, Basel, 2000), pp. 75-105.

[3] J. M. De Koninck and N. Doyon, 'À propos de l'indice de composition des nombres', Monatsh. Math. 139 (2003), 151-167.

[4] J. M. De Koninck and I. Kátai, 'On the mean value of the index of composition', Monatsh. Math. 145 (2005), 131-144.

[5] J. M. De Koninck, I. Kátai and M. V. Subbarao, 'On the index of composition of integers from various sets', Arch. Math. 88 (2007), 524-536.

[6] N. Elkies, 'ABC implies Mordell', Internat. Math. Res. Notices 7 (1991), 99-109.

[7] P. Erdős, 'On the normal number of prime factors of $p-1$ and some other related problems concerning Euler's $\phi$ function’, Quart. J. Math. 6 (1935), 205-213.

[8] A. Granville, 'ABC allows us to count squarefrees', Internat. Math. Res. Notices 19 (1998), 991-1009.

[9] A. Ivić, The Riemann Zeta-Function. Theory and Applications (Dover, Minneola, NY, 2003).

[10] M. Langevin, 'Partie sans facteur carré d'un produit d'entiers voisins', in: Approximations diophantiennes et nombres transcendants (Luminy, 1990) (de Gruyter, Berlin, 1992), pp. 203-214.

[11] F. Luca and C. Pomerance, 'Irreducible radical extensions and Euler function chains', in: Combinatorial Number Theory (Proceedings of the 'Integers Conference 2005' in Celebration of the 70th Birthday of R. Graham) (eds. B. Landman, M. Nathanson, J. Nešetril, R. Nowakowski and C. Pomerance) (de Gruyter, Berlin, 2007), pp. 351-362.

[12] W. Zhai, 'On the mean value of the index of composition of an integer', Acta Arith. 125 (2006), 331-345.

JEAN-MARIE DE KONINCK, Département de mathématiques et de statistique, Université Laval, Québec G1V OA6, Canada

e-mail: jmdk@mat.ulaval.ca

FLORIAN LUCA, Mathematical Institute, UNAM, Ap. Postal 61-3 (Xangari),

CP 58 089, Morelia, Michoacán, Mexico

e-mail: fluca@matmor.unam.mx 